\title{
Moments Based Framework for Performance Analysis of One-Way/Two-Way CSI-Assisted AF Relaying
}

\author{
Minghua Xia, Member, IEEE, and Sonia Aïssa, Senior Member, IEEE
}

\begin{abstract}
When analyzing system performance of conventional one-way relaying or advanced two-way relaying, these two techniques are always dealt with separately and, thus, their performance cannot be compared efficiently. Moreover, for ease of mathematical tractability, channels considered in such studies are generally assumed to be subject to Rayleigh fading or to be Nakagami- $m$ channels with integer fading parameters, which is impractical in typical urban environments. In this paper, we propose a unified moments-based framework for general performance analysis of channel-state-information (CSI) assisted amplify-and-forward (AF) relaying systems. The framework is applicable to both one-way and two-way relaying over arbitrary Nakagami- $m$ fading channels, and it includes previously reported results as special cases. Specifically, the mathematical framework is firstly developed under the umbrella of the weighted harmonic mean of two Gamma-distributed variables in conjunction with the theory of Padé approximants. Then, general expressions for the received signal-to-noise ratios of the users in one-way/twoway relaying systems and the corresponding moments, moment generation function, and cumulative density function are established. Subsequently, the mathematical framework is applied to analyze, compare, and gain insights into system performance of one-way and two-way relaying techniques, in terms of outage probability, average symbol error probability, and achievable data rate. All analytical results are corroborated by simulation results as well as previously reported results whenever available, and they are shown to be efficient tools to evaluate and compare system performance of one-way and two-way relaying.
\end{abstract}

Index Terms-Amplify-and-forward, moments, Nakagami fading, one-way relaying, Padé approximants, performance analysis, two-way relaying, weighted harmonic mean.

\section{INTRODUCTION}

$\mathbf{C}$ OOPERATIVE relaying has shown great potential for next-generation wireless systems, since its significant cooperative diversity gain benefits improving spectral efficiency and extending the coverage of wireless systems without extra transmit power [1]. For the conventional one-way relaying shown in Fig. 1-a), an intermediate relay $\mathrm{R}$ can be exploited to help the source $\mathrm{S}$ forward signals to the destination $\mathrm{D}$ if the direct channel between S and D suffers from deep fading. This one-way relaying scheme involves two consecutive phases for only one data transmission (i.e. $\mathrm{S} \rightarrow \mathrm{R} \rightarrow \mathrm{D}$ ) and, thus, degrades spectral efficiency significantly. In order to improve spectral efficiency, two-way relaying was proposed in [2], where two users $S_{1}$ and $S_{2}$ exchange data simultaneously with

Manuscript received 15 August, 2011; revised 6 March, 2012 and 16 May, 2012. This work was supported by King Abdullah University of Science and Technology (KAUST), Thuwal, Kingdom of Saudi Arabia (KSA).

M. Xia is with the Division of Physical Sciences and Engineering, KAUST, Thuwal, KSA (e-mail: minghua.xia@ieee.org).

S. Aïssa is with Institut National de la Recherche Scientifique (INRS), University of Quebec, Montreal, QC, Canada (e-mail: aissa@emt.inrs.ca).

Digital Object Identifier $* * *$

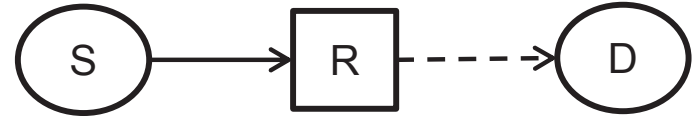

a) One-way relaying

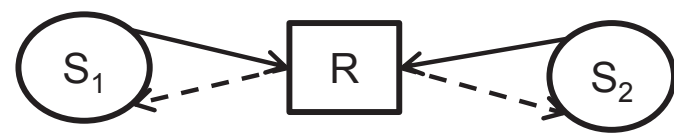

b) Two-way relaying

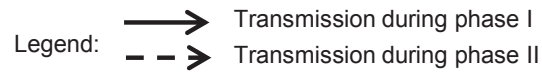

Fig. 1. System model of dual-hop amplify-and-forward relaying.

the aid of the relaying node $\mathrm{R}$, as illustrated in Fig. 1-b). That is, two-way relaying completes two data transmissions (i.e. $\mathrm{S}_{1} \rightarrow \mathrm{R} \rightarrow \mathrm{S}_{2}$ and $\mathrm{S}_{2} \rightarrow \mathrm{R} \rightarrow \mathrm{S}_{1}$ ) during two phases and, hence, it achieves higher spectral efficiency than one-way relaying while using the same amount of system resources.

For the relaying node in either one-way or two-way relaying systems, there are mainly two relaying techniques, namely, decode-and-forward (DF) and amplify-and-forward (AF). In contrast to DF relaying, AF relaying does not require decoding operation and it simply amplifies the received signal prior to transmission. That is, AF relaying is transparent to the modulation and coding schemes of the source and, therefore, it has a simple structure and can be more easily deployed in practical applications.

In the $\mathrm{AF}$ technique, there are primarily three strategies to determine the relay gain. The first strategy, referred to as blind relay, fixes the relay gain regardless of the channel fluctuations. Its performance deteriorates if the channel amplitude varies a lot. The second strategy uses statistical channel state information (CSI) of the channels and is generally called the semi-blind relay. The third strategy, on the other hand, exploits full CSI of the channels during the first phase to determine the relay gain. This CSI-assisted approach outperforms the blind and semi-blind ones and serves as benchmark for performance evaluation of various relaying systems.

When analyzing system performance of CSI-assisted oneway or two-way AF relaying, in order to obtain closed-form expressions for the considered metrics, it is generally assumed that the channels are subject to Rayleigh fading [1]-[7]. However, it is well known that Nakagami- $m$ fading is more general and it can model extensive fading scenarios. When Nakagami$m$ fading is concerned, due to the difficulty of mathematical 
tractability, it is mostly supposed that the Nakagami fading parameters take integer values, see for example [8], [9] for studies dealing with one-way relaying and [10], [11] for twoway relaying. The scenario with integer fading parameters is a very particular case. In reality, on the other hand, propagation environments where the fading parameter $m$ takes non-integer values are very common, for instance, micro-cellular scenarios with specular components and land mobile satellite channels [12], [13]. More specifically, the field measurements in urban scenarios demonstrate that "the most likely value of $m$ oscillates between 1 and 2 " and that "the average estimated value of $m$ is 1.56 " [12]. Therefore, accurate performance evaluation of one-way/two-way CSI-assisted AF relaying over general Nakagami- $m$ fading channels becomes extremely important for practical purposes.

For one-way and two-way CSI-assisted AF relaying systems under study in this paper, exact performance analysis over general Nakagami- $m$ fading channels is extremely complicated, if not impossible. Even for simpler one-way semi-blind relaying systems, exact performance analysis is highly mathematically involved, as recently reported by the same authors in [14]. Therefore, general performance analysis of both relaying systems under study was hardly touched upon and only some very special cases were dealt with and scattered in the open literature. For example, provided that the channel fading at two consecutive hops is symmetric (i.e. $m_{1}=m_{2}$ ) and the average signal-to-noise ratios (SNRs) of the hops are identical (i.e. $\bar{\gamma}_{1}=\bar{\gamma}_{2}$ ), outage probability and average bit error rate of one-way CSI-assisted AF relaying over general Nakagami$m$ fading channels were investigated in [15]. Performance bounds on average symbol error probability were studied in [16], [17]. For two-way relaying, the methodology in [15] was also used in [18], where symmetric fading scenarios were still considered and the performance analysis was further restricted to Rayleigh fading (i.e. $m_{1}=m_{2}=1$ ).

In all the above mentioned works, one-way and two-way relaying were always dealt with separately and, thus, their system performance cannot be compared efficiently. In addition, due to the extreme difficulty of mathematical tractability, comprehensive performance analysis of CSI-assisted AF relaying over general Nakagami- $m$ channels was never reported before, to the best of the authors' knowledge. With these regards, this paper proposes a unified framework for the comprehensive performance analysis of CSI-assisted one-way and twoway relaying, by means of the moments-based approach in conjunction with the technique of Padé approximants (more details on the rationale and application of these two methods in the open literature can be found in Remarks 1 and 2 of Sections II-A and II-B, respectively).

The proposed framework is applicable to both one-way and two-way relaying schemes considering Nakagami- $m$ channels with arbitrary fading parameters and, hence, it can be used to compare system performance of both schemes efficiently. Given its generality, this framework also includes previously reported results as special cases. Moreover, the framework can be extended to other related areas, such as the dual-hop relaying scenario with optimal power allocation and the scenario of multi-hop relaying. Specifically, our main contributions are three-fold:
1) We propose a general mathematical model for the received SNRs of the users in one-way and two-way CSIassisted AF relaying systems, under the umbrella of the weighted harmonic mean of two Gamma random variables. Based on this model, the moments of the received SNRs are developed.

2) With the moments of the received SNRs, the theory of Padé approximants is exploited to derive the SNRs' moment generation function (MGF) in a simple and analytical form. In particular, an exact MGF of the received SNRs is also derived in closed-form and it is used to explicitly measure the relative error of the Padé approximant, which is critical to determining the orders of polynomials involved in the Padé approximant. With the analytical Padé approximant to MGF, the cumulative density function (CDF) of the received SNRs is also obtained.

3) After obtaining the general expressions for the moments, the MGF, and the CDF of the received SNRs, three important performance measures of one-way and twoway relaying are investigated, namely, outage probability, average symbol error probability (ASEP), and achievable data rate. Furthermore, thanks to the generality of the proposed framework, these two relaying techniques are efficiently compared and some insights into system performance are finally gained.

In detailing these contributions, the rest of this paper is organized as follows. Section II develops the mathematical statistics relevant to the weighted harmonic mean of two Gamma-distributed random variables, including the moments, the MGF, and the CDF. Section III describes system model of one-way and two-way relaying and, in particular, the general expressions for the received SNRs and their corresponding moments, MGF, and CDF are explicitly established. Section IV is devoted to the application of the preceding mathematical statistics to the performance analysis and comparison of the one-way and two-way relaying systems under study and, finally, concluding remarks are provided in Section V.

\section{Mathematical Statistics: Weighted Harmonic MEAN OF TWO GAMMA VARIABLES}

Given two numbers $X_{1}$ and $X_{2}$, their weighted harmonic mean, $H_{w}\left(X_{1}, X_{2}\right)$, is defined as [19, p. 4]:

$$
\begin{aligned}
H_{w}\left(X_{1}, X_{2}\right) & \triangleq\left[\frac{1}{a+b}\left(\frac{a}{X_{1}}+\frac{b}{X_{2}}\right)\right]^{-1} \\
& =\frac{(a+b) X_{1} X_{2}}{a X_{2}+b X_{1}}
\end{aligned}
$$

where the subscript " $w$ " of $H_{w}\left(X_{1}, X_{2}\right)$ stands for the "weighted version" of the harmonic mean of $X_{1}$ and $X_{2}$ with weights $a$ and $b$, respectively. In particular, when the weights $(a, b)=(1,1)$, the weighted harmonic mean of $X_{1}$ and $X_{2}$ reduces to the conventional harmonic mean defined as [20, Eq. (1.2.19)]:

$$
\begin{aligned}
H\left(X_{1}, X_{2}\right) & \triangleq\left[\frac{1}{2}\left(\frac{1}{X_{1}}+\frac{1}{X_{2}}\right)\right]^{-1} \\
& =\frac{2 X_{1} X_{2}}{X_{1}+X_{2}}
\end{aligned}
$$


On the other hand, it is well known that a random variable $X$ has a Gamma distribution if its probability density function (PDF) is given by [21, Eq. (20.6)]

$$
f_{X}(x)=\frac{x^{\alpha-1}}{\beta^{\alpha} \Gamma(\alpha)} e^{-\frac{x}{\beta}}, \quad x \geq 0
$$

where $\alpha, \beta>0$ denote the shape parameter and scaling factor, respectively, and where $\Gamma($.$) is the Gamma function [20, Eq.$ (5.2.1)]. For the sake of notational convenience, we use the notation $X \sim G(\alpha, \beta)$ to represent a random variable with PDF (3).

\section{A. The Moments of $H_{w}\left(X_{1}, X_{2}\right)$}

When $X_{1}$ and $X_{2}$ are two independent but not necessarily identically distributed (i.n.i.d) Gamma random variables, that is, $X_{i} \sim G\left(\alpha_{i}, \beta_{i}\right), i=1,2$, the moments of their weighted harmonic mean are as stated in the following theorem.

Theorem 1: (Moments of the Weighted Harmonic Mean of Two Gamma Variables) Let $X_{1}$ and $X_{2}$ be two i.n.i.d Gamma random variables with parameters $\alpha_{i}$ and $\beta_{i}$, that is, $X_{i} \sim G\left(\alpha_{i}, \beta_{i}\right), i=1,2$. Then, the $n^{\text {th }}$-order moment of their weighted harmonic mean is given by Eq. (4) at the top of the next page, where the operator $\mathbb{E}\{$.$\} denotes$ statistical expectation, $B(x, y)$ refers to the Beta function [22, Eq. (8.384.1)] and ${ }_{2} F_{1}(a, b ; c ; x)$ stands for the Gaussian hypergeometric function [22, Eq. (9.100)], and where

$$
\alpha \triangleq \begin{cases}\alpha_{2}, & \text { if } b \beta_{1} \leq a \beta_{2} \\ \alpha_{1}, & \text { otherwise }\end{cases}
$$

and

$$
\beta_{\text {min }} \triangleq \min \left(b \beta_{1}, a \beta_{2}\right), \quad \beta_{\max } \triangleq \max \left(b \beta_{1}, a \beta_{2}\right) .
$$

Proof: See Appendix A.

For the ease of subsequent applications, the first-order and second-order moments of the weighted harmonic mean of two Gamma random variables are explicitly expressed in the following corollary.

Corollary 1: The first-order and second-order moments of the weighted harmonic mean of two i.n.i.d Gamma variables, $X_{i} \sim G\left(\alpha_{i}, \beta_{i}\right), i=1,2$, are given by Eqs. (7) and (8) at the top of the next page, respectively.

Proof: The results shown in (7)-(8) can be proved by substituting $n=1$ and $n=2$ into (4), respectively, and using the recurrence formula of the Gamma function, that is, $\Gamma(x+$ $1)=x \Gamma(x)$ [20, Eq. (5.5.1)], as well as some straightforward algebraic manipulations.

Special Cases: When the weights of the variables $X_{1}$ and $X_{2}$ are unity, i.e. when $(a, b)=(1,1)$, then by the definitions (1)-(2), the weighted harmonic mean of $X_{1}$ and $X_{2}$ reduces to their conventional harmonic mean. Furthermore, when $X_{1}$ and $X_{2}$ are Gamma-distributed, we have

1) If $X_{1}$ and $X_{2}$ are independent and identically distributed (i.i.d.) Gamma variables, i.e. $X_{i} \sim G(\alpha, \beta), i=1,2$, then recalling the fact that ${ }_{2} F_{1}(a, b ; c ; 0)=1$ [20, Eq. (15.2.1)], the $n^{\text {th }}$-order moment of the harmonic mean of $X_{1}$ and $X_{2}$ given by (4) reduces to

$$
\mathbb{E}\left\{H^{n}\right\}=B(n+\alpha, n+\alpha) \frac{(2 \beta)^{n} \Gamma(n+2 \alpha)}{\Gamma^{2}(\alpha)}
$$

$$
=\left(\frac{\beta}{2}\right)^{n} \frac{(\alpha)_{n}(2 \alpha)_{n}}{\left(\alpha+\frac{1}{2}\right)_{n}},
$$

where the duplication formula of the Gamma function $\Gamma(2 x)=\pi^{-\frac{1}{2}} 2^{2 x-1} \Gamma(x) \Gamma\left(x+\frac{1}{2}\right)$ [20, Eq. (5.5.5)] was used to derive (9) with $(x)_{n}=\Gamma(x+n) / \Gamma(x)$ being the Pochhammer's symbol [20, Eq. (5.2.5)]. The expression (9) is in agreement with the previously reported result in [15, Eq. (7)].

2) If $X_{1}$ and $X_{2}$ have the same shape parameters but different scaling factors, i.e. $X_{i} \sim G\left(\alpha, \beta_{i}\right), i=1,2$, the expression in (4) reduces to Eq. (10) at the top of the next page, which is consistent with the previously reported result in [18, Eq. (2)].

Remark 1: The moments of a random variable are very important statistics and they can be widely applied in practice. For instance, in one-way AF relaying systems, the first and second order moments of received SNR were used to efficiently evaluate achievable data rate [14], [23]. On the other hand, the PDF of a random variable can be determined or approximated from a finite number of its moments. For instance, the well-known Pearson system of distributions involves only the first four moments [24, Chap. 6] and it finds many applications in wireless systems, see for example [25]. More techniques on approximating a PDF from its moments can be found in [24, Chap. 6], [26, Chap. 8]. In the next subsection, in order to exploit more powerful MGF-based methodology to analyze system performance, we derive the MGF from the above obtained moments.

\section{B. The $M G F$ of $H_{w}\left(X_{1}, X_{2}\right)$}

In this subsection, we first derive the exact MGF of $H_{w}\left(X_{1}, X_{2}\right)$ in closed-form. Due to its high complexity, it is hard to be further processed and, thus, we then develop an approximate MGF in a simple and analytical form, based on the theory of Padé approximants [28].

The exact closed-form MGF of the weighted harmonic mean of two i.n.i.d Gamma random variables is summarized in the following theorem.

Theorem 2: (MGF of the Weighted Harmonic Mean of Two Gamma Variables) The MGF of the weighted harmonic mean shown in (1) of two i.n.i.d Gamma variables, $X_{i} \sim G\left(\alpha_{i}, \beta_{i}\right)$, $i=1,2$, is given by

$$
\mathrm{M}_{H_{w}}(s)=1-\frac{s k_{1}^{\alpha_{1}}}{\Gamma\left(\alpha_{1}\right) \Gamma\left(\alpha_{2}\right) k_{2}^{\alpha_{1}+2}}\left[A\left(\alpha_{1}\right)+A\left(-\alpha_{1}\right)\right]
$$

where the constants $k_{1}$ and $k_{2}$ are given by

$$
k_{1} \triangleq \sqrt{\frac{a}{(a+b) \beta_{1}}}, k_{2} \triangleq \sqrt{\frac{b}{(a+b) \beta_{2}}},
$$

and where the function $A(x)$ is defined by Eq. (13) shown in the next page, with $F_{4}($.$) denoting the fourth Appell gener-$ alized bivariate hypergeometric function [20, Eq. (16.13.4)].

Proof: See Appendix B.

It is noteworthy that the Appell function $F_{4}($.$) involved$ in (13) can be easily computed by the built-in function appellf4(.) of the up-to-date Python package MPMATH [30], although it is not yet implemented in popular mathematical 


$$
\begin{aligned}
\mathbb{E}\left\{H_{w}^{n}\right\}= & B\left(n+\alpha_{1}, n+\alpha_{2}\right) \frac{\Gamma\left(n+\alpha_{1}+\alpha_{2}\right)}{\Gamma\left(\alpha_{1}\right) \Gamma\left(\alpha_{2}\right)}\left(\frac{a+b}{a b} \beta_{\min }\right)^{n}\left(\frac{\beta_{\min }}{\beta_{\max }}\right)^{\alpha} \\
& \times{ }_{2} F_{1}\left(n+\alpha, n+\alpha_{1}+\alpha_{2} ; 2 n+\alpha_{1}+\alpha_{2} ; 1-\frac{\beta_{\min }}{\beta_{\max }}\right), \quad n \geq 0 .
\end{aligned}
$$

$$
\mathbb{E}\left\{H_{w}\right\}=\frac{\alpha_{1} \alpha_{2}(a+b) \beta_{\min }^{\alpha+1}}{a b\left(1+\alpha_{1}+\alpha_{2}\right) \beta_{\max }^{\alpha}}{ }_{2} F_{1}\left(1+\alpha, 1+\alpha_{1}+\alpha_{2} ; 2+\alpha_{1}+\alpha_{2} ; 1-\frac{\beta_{\min }}{\beta_{\max }}\right) .
$$

$$
\mathbb{E}\left\{H_{w}^{2}\right\}=\frac{\alpha_{1} \alpha_{2}\left(1+\alpha_{1}\right)\left(1+\alpha_{2}\right)(a+b)^{2} \beta_{\min }^{\alpha+2}}{\left(2+\alpha_{1}+\alpha_{2}\right)\left(3+\alpha_{1}+\alpha_{2}\right)(a b)^{2} \beta_{\max }^{\alpha}}{ }_{2} F_{1}\left(2+\alpha, 2+\alpha_{1}+\alpha_{2} ; 4+\alpha_{1}+\alpha_{2} ; 1-\frac{\beta_{\min }}{\beta_{\max }}\right) .
$$

$$
\mathbb{E}\left\{H^{n}\right\}=B(n+\alpha, n+\alpha) \frac{2^{n} \Gamma(n+2 \alpha) \beta_{\min }^{\alpha+n}}{\Gamma^{2}(\alpha) \beta_{\max }^{\alpha}} F_{1}\left(n+\alpha, n+2 \alpha ; 2 n+2 \alpha ; 1-\frac{\beta_{\min }}{\beta_{\max }}\right)
$$

$$
A(x)=\left(\frac{k_{1}}{k_{2}}\right)^{x} \Gamma(-x) \Gamma(x+1) \Gamma\left(x+\alpha_{2}+1\right) F_{4}\left(x+1 ; x+\alpha_{2}+1 ; 2, x+1 ;-\frac{s}{k_{2}^{2}},\left(\frac{k_{1}}{k_{2}}\right)^{2}\right)
$$

softwares, such as Matlab, Mathematica and Maple. As such, the MGF given by (11) can be efficiently evaluated in a numerical way. However, due to the Appell function $F_{4}($. involved, the MGF in (11) cannot be easily manipulated for further derivations. In order to proceed, we develop an approximate MGF in a simple and analytical form as follows.

By definition, the MGF of the weighted harmonic mean of $X_{1}$ and $X_{2}, H_{w}\left(X_{1}, X_{2}\right)$, is expressed as

$$
\begin{aligned}
\mathrm{M}_{H_{w}}(s) & =\mathbb{E}\left\{\exp \left(-s H_{w}\right)\right\} \\
& =\mathbb{E}\left\{\sum_{n=0}^{\infty} \frac{(-1)^{n}}{n !} H_{w}^{n} s^{n}\right\} \\
& =\sum_{n=0}^{\infty} c_{n} s^{n},
\end{aligned}
$$

where the Maclaurin series expansion of the exponential function was exploited to derive (14) and, for notational convenience, parameter $c_{n}$ was introduced in (15) according to the following definition

$$
c_{n} \triangleq \frac{(-1)^{n}}{n !} \mathbb{E}\left\{H_{w}^{n}\right\}, \quad n \geq 0
$$

where $\mathbb{E}\left\{H_{w}^{n}\right\}$ denotes the $n^{\text {th }}$-order moment of $H_{w}\left(X_{1}, X_{2}\right)$. Since the values of the closed-form expression of $\mathbb{E}\left\{H_{w}^{n}\right\}$ given in (4) are finite for any $n \geq 0$, then by use of the ratio test, the associated positive series of the alternating series in (15) converges absolutely for all finite $s$, i.e. $|s|<\infty$. Therefore, the alternating series in (15) converges absolutely for $|s|<\infty$. Notice that, the exact value of the infinite series (15) can be readily obtained by numerically computing the closed-form expression (11).

Although the infinite series (15) is absolutely convergent, it converges slowly and has to be truncated for practical computation. In particular, if (15) is truncated after $N+1$ terms, then

$$
\mathrm{M}_{H_{w}}(s) \approx \sum_{n=0}^{N} c_{n} s^{n}+O\left(s^{N+1}\right),
$$

where $O($.$) refers to the Landau notation [20, Eq. (2.1.3)] and$ $O\left(s^{N+1}\right)$ stands for the truncation error taking into account terms of higher order than $s^{N}$. Therefore, we need to find the "best" approximation to $\mathrm{M}_{H_{w}}(s)$ in the sense that the truncation error is as small as possible. An approximation of this type is known as Padé approximant [28]. More specifically, according to the well-known Baker's definition [28, p. 21], the rational function defined as the ratio of two ordinary polynomials of orders $p$ and $q$, respectively:

$$
\widetilde{\mathrm{M}}_{H_{w}}(s) \triangleq \frac{\sum_{k=0}^{p} a_{k} s^{k}}{1+\sum_{k=1}^{q} b_{k} s^{k}}
$$

is said to be a Padé approximant to $\mathrm{M}_{H_{w}}(s)$, if $\mathrm{M}_{H_{w}}(s)-$ $\widetilde{\mathrm{M}}_{H_{w}}(s)=O\left(s^{p+q+1}\right)$. Conventionally, the coefficients $a_{k}$, $k=0, \cdots, p$ and $b_{k}, k=0, \cdots, q$ in (18) can be uniquely determined by solving the so-called Padé equations [31, Eqs. (4)-(5), p. 75]. However, when the polynomials are of high orders $(p, q)$, solving the Padé equations is very tedious. A more systematic procedure can be developed by using the Nuttall's compact form [28, p. 17]. Explicitly, the Padé approximant (18) can be expressed as

$$
\widetilde{\mathrm{M}}_{H_{w}}(s)=\sum_{n=0}^{p-q} c_{n} s^{n}+\left\{\boldsymbol{c}^{T}[\boldsymbol{W}(s)]^{-1} \boldsymbol{c}\right\} s^{p-q+1},
$$

where the operators $(.)^{T}$ and $(.)^{-1}$ denote matrix transpose and inverse, respectively, and where

$$
\boldsymbol{c}^{T}=\left[\begin{array}{llll}
c_{p-q+1} & c_{p-q+2} & \cdots & c_{p}
\end{array}\right]_{1 \times q},
$$


$\boldsymbol{W}(s)$ is expressed as Eq. (21) at the top of the next page, and $c_{n}$ is given by (16) when $n \geq 0$ and $c_{n} \equiv 0$ if $n<0$. Consequently, in view of (4), (16), and (19)-(21), the Padé approximant (18) can be readily obtained.

The orders $(p, q)$ of the numerator and denominator polynomials in the Padé approximant shown in (19) can be determined as follows. According to [32], the best value of $q$ should be the number of already known coefficients of the Maclaurin series in (15). On the other hand, the order of the numerator polynomial should be no larger than the order of the denominator polynomial, that is, $p \leq q$, since it is only for such Padé approximants that the convergence rate and uniqueness can be guaranteed [28]. As a rule of thumb, the first sub-diagonal Padé approximant with $p=q-1$ is more accurate than the others and it is extensively applied in practice [32]-[36].

In order to measure the accuracy of the Pade approximant (19) to its exact value given by (11), we define the relative error as

$$
\xi(s) \triangleq\left|\frac{\mathrm{M}_{H_{w}}(s)-\widetilde{\mathrm{M}}_{H_{w}}(s)}{\mathrm{M}_{H_{w}}(s)}\right| .
$$

Figure 2 illustrates the effectiveness of the Padé approximant (19), by comparing its numerical results with its corresponding exact values computed by (11). In the simulations, the parameters of two Gamma variables involved are $\left(\alpha_{1}, \beta_{1}\right)=(1.3,1),\left(\alpha_{2}, \beta_{2}\right)=(2.5,1)$ and their weights $(a, b)=(2,1)$. In addition, the variable $s$ of the Padé approximant (19) and the exact MGF expression (11) takes values from 0 to 2 with step size 0.2 . This is because, by the final value theorem for Laplace transforms, the region $s \rightarrow 0$ in the MGF $M_{H_{w}}(s)$ corresponds to the region $x \rightarrow \infty$ in its corresponding PDF $f_{H_{w}}(x)$. This region reflects the right tail of the PDF and it is the most important region in practical applications [37, p. 55]. From the left-hand side panel of Fig. 2, it is observed that the Padé approximant with orders $(p, q)=(7,8)$ coincides very well with the exact MGF in the region of $0 \leq s<1$. The right-hand side panel of Fig. 2 shows that the relative error is smaller than $10^{-2}$ in this region of $s$. Furthermore, it is seen that the Padé approximant becomes more and more accurate even in a larger region as the values of orders $(p, q)$ increase.

Remark 2: Padé approximant is a powerful technique to represent a function through the ratio of two ordinary polynomials. In particular, if the Maclaurin series expansion of a specific function exists, its Padé approximant can always be found by solving the Padé equations. Moreover, the Padé approximant usually outperforms the truncated Maclaurin series approximation in terms of truncation error [31, Section 10.3]. For signal processing and performance analysis of communication systems, Padé approximant has found many applications, see for example [32]-[36]. However, unlike our Theorem 2, it is generally hard to find the exact MGF expression and, thus, the relative error of Padé approximant cannot be explicitly measured by (22). This hinders determining the orders of polynomials involved in Padé approximant and, thus, some other indirect reference has to be resorted to, see for example [33], [34]. For more details about Padé approximant, the interested reader is referred to the seminal encyclopedic

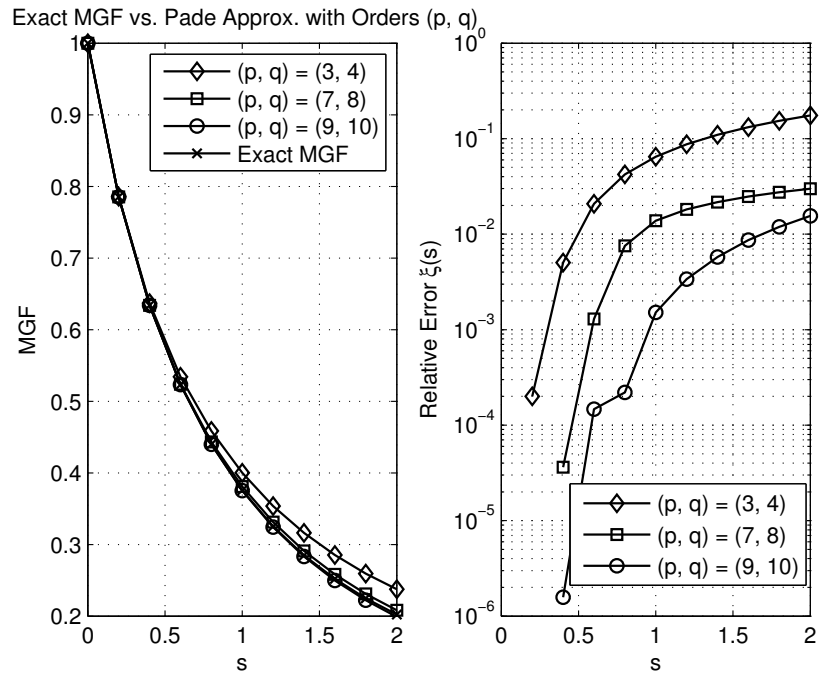

Fig. 2. The effectiveness of Padé approximants.

monograph [28].

\section{The CDF of $H_{w}\left(X_{1}, X_{2}\right)$}

Applying the partial fraction expansion method to the Padé approximant (18), we get

$$
\widetilde{\mathrm{M}}_{H_{w}}(s)=\sum_{k=1}^{q} \frac{\nu_{k}}{s+\mu_{k}},
$$

where $\nu_{k}$ and $-\mu_{k}, k=1, \cdots, q$, are the residues and the poles of $\widetilde{\mathrm{M}}_{H_{w}}(s)$, respectively. Then, applying the inverse Laplace transformation to (23), the $\mathrm{CDF}$ of $H_{w}\left(X_{1}, X_{2}\right)$ can be obtained according to:

$$
\begin{aligned}
F_{H_{w}}(x) & =\mathcal{L}^{-1}\left\{\frac{1}{s} \widetilde{\mathrm{M}}_{H_{w}}(s)\right\} \\
& =1-\sum_{k=1}^{q} \frac{\nu_{k}}{\mu_{k}} \exp \left(-\mu_{k} x\right),
\end{aligned}
$$

where the operator $\mathcal{L}^{-1}\{$.$\} stands for the inverse Laplace$ transformation.

Notice that, in the applications developed later on in this paper, the orders $(p, q)$ involved in the Padé approximant (19) are chosen to guarantee that the relative error $\xi(s)$ defined in (22) at $s=1$ is no larger than $10^{-2}$, i.e. $\xi(1) \leq 10^{-2}$. The region $0 \leq s<1$ lies inside the unit circle in the complex frequency domain and determines the right tail of the corresponding PDF in the time domain. This small relative error ensures that the Padé approximant reflects its corresponding exact MGF accurately. In such case, the value of the order $q$ ranges from 2 to 7 and, hence, the $q \times q$ dimensional matrix inversion involved in (19) can be easily computed. Furthermore, the numerical results of the outage probability evaluated by (24) match at the $4^{\text {th }}$ significant digit those evaluated by the exact expressions (36) and (38), which will be discussed further in Section IV-A below. 


$$
\boldsymbol{W}(s)=\left[\begin{array}{cccc}
c_{p-q+1}-s c_{p-q+2} & c_{p-q+2}-s c_{p-q+3} & \cdots & c_{p}-s c_{p+1} \\
c_{p-q+2}-s c_{p-q+3} & c_{p-q+3}-s c_{p-q+4} & \cdots & c_{p+1}-s c_{p+2} \\
\vdots & \vdots & \ddots & \vdots \\
c_{p}-s c_{p+1} & c_{p+1}-s c_{p+2} & \cdots & c_{p+q-1}-s c_{p+q}
\end{array}\right]_{q \times q}
$$

\section{System Model of DuAL-Hop CSI-Assisted AF RELAYING}

In this section, we first concisely describe the system model of one-way and two-way AF relaying. Then, the general expressions for the received SNRs and their corresponding moments, MGF, and CDF are established.

\section{A. System Model}

1) One-Way Relaying: Fig. 1-a) depicts the system model of one-way relaying with single AF relay. The transmission period from the source $S$ to the destination $D$ consists of two consecutive phases. During the first transmission phase, the source transmits the signal to the relay R. During the second phase, the source keeps silent while the CSI-assisted relay amplifies its received signal and forwards it to the destination. Let the instantaneous SNRs at two consecutive hops be $\gamma_{1}$ $(\mathrm{S} \rightarrow \mathrm{R})$ and $\gamma_{2}(\mathrm{R} \rightarrow \mathrm{D})$, respectively. Then, the end-to-end SNR from the source to the destination via the relay $(\mathrm{S} \rightarrow$ $\mathrm{R} \rightarrow \mathrm{D})$ is as given in [3], [8], [9], [15], [17], namely,

$$
\gamma_{\mathrm{D}}=\frac{\gamma_{1} \gamma_{2}}{\gamma_{1}+\gamma_{2}}
$$

where the subscript " $D$ " of $\gamma_{D}$ refers to the destination $D$ in one-way relaying system.

2) Two-Way Relaying: In the two-way relaying system shown in Fig. 1-b), one data exchange between the users $\mathrm{S}_{1}$ and $\mathrm{S}_{2}$ is performed with the assistance of the relay $\mathrm{R}$ during two consecutive phases: multi-access (MAC) phase and broadcast (BC) phase. During the MAC phase, $\mathrm{S}_{1}$ and $\mathrm{S}_{2}$ simultaneously transmit signals to the relay. During the $\mathrm{BC}$ phase, the CSI-assisted relay amplifies its received signal and broadcasts it to both users. With the principle of channel reciprocity over flat fading, the SNR at the forward link $\left(\mathrm{S}_{1} \rightarrow \mathrm{R}\right)$ is identical to that at the backward link $\left(\mathrm{R} \rightarrow \mathrm{S}_{1}\right)$, which is denoted by $\gamma_{1}$ as defined above in one-way relaying system. Similarly, the SNR at the link between $R$ and $S_{2}$ is denoted by $\gamma_{2}$ as defined above. Then, the received SNRs of the users $S_{1}$ and $S_{2}$ are respectively given by [2], [10], [18]

$$
\gamma_{\mathrm{s}_{1}}=\frac{\gamma_{1} \gamma_{2}}{2 \gamma_{1}+\gamma_{2}}, \quad \gamma_{\mathrm{S}_{2}}=\frac{\gamma_{1} \gamma_{2}}{\gamma_{1}+2 \gamma_{2}} .
$$

It is assumed that the channels at the two hops are subject to Nakagami- $m$ fading with fading shape parameters $m_{1}, m_{2} \geq 0.5$, respectively. Consequently, the instantaneous SNR pertaining to each hop is of Gamma distribution such that $\gamma_{i} \sim G\left(m_{i}, \frac{\bar{\gamma}_{i}}{m_{i}}\right), i=1,2$, where $\bar{\gamma}_{i}$ denotes the average SNR. Unlike the open literature [15], [16], [18], the framework proposed in this paper applies to arbitrary Nakagami- $m$ fading scenarios without any limitation on the values of $m_{1}, m_{2}, \bar{\gamma}_{1}$, and $\bar{\gamma}_{2}$.

\section{B. General Expressions for the Received SNRs and Their Related Statistics}

Recalling the definition (1) of the weighted harmonic mean of two variables, it is clear that the received SNRs in (25) and (26) can be expressed in a general form:

$$
\gamma_{j}=\frac{1}{a_{j}+b_{j}} H_{w}\left(\gamma_{1}, \gamma_{2}\right), \quad j \in\left\{\mathrm{D}, \mathrm{S}_{1}, \mathrm{~S}_{2}\right\}
$$

where the weights $\left(a_{\mathrm{D}}, b_{\mathrm{D}}\right)=(1,1),\left(a_{\mathrm{S}_{1}}, b_{\mathrm{S}_{1}}\right)=(1,2)$, and $\left(a_{\mathrm{S}_{2}}, b_{\mathrm{S}_{2}}\right)=(2,1)$. Therefore, based on the mathematical statistics (4), (11), and (24) developed in Section II, the $n^{\text {th }}$ order moment, the MGF, and the CDF of $\gamma_{j}$ can be shown to be expressed as

$$
\begin{gathered}
\mathbb{E}\left\{\gamma_{j}^{n}\right\}=\frac{\mathbb{E}\left\{H_{w}^{n}\right\}}{\left(a_{j}+b_{j}\right)^{n}}, \\
\mathrm{M}_{\gamma_{j}}(s)=\mathrm{M}_{H_{w}}\left(\frac{s}{a_{j}+b_{j}}\right)
\end{gathered}
$$

and

$$
F_{\gamma_{j}}(x)=F_{H_{w}}\left(\left(a_{j}+b_{j}\right) x\right)
$$

respectively.

Besides the one-way and two-way relaying under study in this paper, thanks to the generality of the proposed framework, it can be applied in other related areas. For illustrative purposes, the following remarks outline two possible extensions in relaying systems.

Remark 3: (Extension to the dual-hop relaying scenario with optimal power allocation) In the system model described in Section III-A, all source/relaying nodes use the same transmit power levels. If different nodes have different transmit powers, on the other hand, the only difference in the ensuing received SNRs lies in the coefficients of $\gamma_{1}$ and $\gamma_{2}$ in the denominators of (25) and (26). In such case, the general expressions (27)-(30) still hold.

Remark 4: (Extension to the scenario of multi-hop relaying) In the present paper, our focus is on dual-hop relaying. Nevertheless, the proposed moments-based methodology can be extended to analyze multi-hop CSI-assisted relaying. Indeed, in multi-hop scenario, the end-to-end SNR is still determined by the harmonic mean of the SNRs at the individual hops, and a similar application in multi-hop blind relaying can be found in [34].

\section{Applications: System Performance Analysis}

Having developed the general expressions for the received SNRs and their related statistics, we now apply these general statistical expressions (28)-(30) to analyze and compare system performance of one-way and two-way CSI-assisted AF relaying, in terms of outage probability, ASEP, and achievable data rate. 


\section{A. Outage Probability}

As an important performance measure of wireless communication systems, outage probability is defined as the probability that the instantaneous received SNR falls below a predefined threshold $\gamma_{\mathrm{th}}$. This SNR threshold guarantees the minimum quality-of-service (QoS) requirement of served users. By definition, evaluating the CDF (30) of the received SNR at $\gamma_{j}=\gamma_{\text {th }}$, we immediately obtain the outage probability of user $j$. Mathematically, the outage probability of user $j$, $j \in\left\{\mathrm{D}, \mathrm{S}_{1}, \mathrm{~S}_{2}\right\}$, is given by

$$
\begin{aligned}
P_{j}\left(\gamma_{\mathrm{th}}\right) & =\operatorname{Pr}\left\{\gamma_{j}<\gamma_{\mathrm{th}}\right\} \\
& =F_{\gamma_{j}}\left(\gamma_{\mathrm{th}}\right) .
\end{aligned}
$$

For the one-way relaying system under study, there is only one destination and, thus, its outage probability is identical to the outage probability of the destination. That is,

$$
P_{1 \text { way }}^{\text {outage }}\left(\gamma_{\text {th }}\right)=F_{\mathrm{D}}\left(\gamma_{\text {th }}\right)
$$

where the subscript "1way" of $P_{1 \text { way }}^{\text {outage }}\left(\gamma_{\text {th }}\right)$ stands for the one-way relaying system.

For the two-way relaying system, there are two users $\mathrm{S}_{1}$ and $\mathrm{S}_{2}$ exchanging data simultaneously. Therefore, unlike oneway relaying, there are two different ways to define the outage probability of two-way relaying. One is defined as the mean of the outage probabilities of both users, and the other is defined as the probability that either $S_{1}$ or $S_{2}$ suffers outage. Clearly, the latter is more stringent than the former. Notice that the outage events $\gamma_{\mathrm{S}_{1}}<\gamma_{\text {th }}$ and $\gamma_{\mathrm{S}_{2}}<\gamma_{\text {th }}$ are dependent, since both $\gamma_{\mathrm{S}_{1}}$ and $\gamma_{\mathrm{S}_{2}}$ are functions of $\gamma_{1}$ and $\gamma_{2}$. Therefore, the outage probability of two-way relaying system is given by

$$
\begin{aligned}
P_{2 \text { way }}^{\text {outage }}\left(\gamma_{\text {th }}\right) \triangleq & \operatorname{Pr}\left\{\gamma_{\mathrm{S}_{1}}<\gamma_{\text {th }} \text { or } \gamma_{\mathrm{S}_{2}}<\gamma_{\text {th }}\right\} \\
\leq & F_{\mathrm{S}_{1}}\left(\gamma_{\mathrm{th}}\right)+F_{\mathrm{S}_{2}}\left(\gamma_{\mathrm{th}}\right) \\
& -F_{\mathrm{S}_{1}}\left(\gamma_{\mathrm{th}}\right) F_{\mathrm{S}_{2}}\left(\gamma_{\mathrm{th}}\right)
\end{aligned}
$$

where the subscript "2way" of $P_{2 \text { way }}^{\text {outage }}\left(\gamma_{\text {th }}\right)$ refers to the twoway relaying system. Furthermore, when the channel fading at two consecutive hops are non-symmetric (i.e. their fading parameters $m_{1}$ and $m_{2}$ are different), the outage probabilities of users $S_{1}$ and $S_{2}$ are non-symmetric and, thus, (34) can be approximately given by

$$
P_{2 \text { way }}^{\text {outage }}\left(\gamma_{\text {th }}\right) \approx \max \left\{F_{\mathrm{S}_{1}}\left(\gamma_{\mathrm{th}}\right), F_{\mathrm{S}_{2}}\left(\gamma_{\mathrm{th}}\right)\right\} .
$$

For the purposes of comparison, the previously reported expressions for the outage probability of one-way and two-way relaying under the particular scenario with integer Nakagami$m$ fading parameters, are briefly reproduced below. ${ }^{1}$ Specifically, for one-way relaying, the exact outage probability under the scenario with integer fading parameters $m_{1}$ and $m_{2}$ is shown as Eq. (36) at the top of the next page [9, Eq. (2)], where the parameter $C(n, k, l)$ is defined as Eq. (37) at the top of the next page. On the other hand, for user $\mathrm{S}_{1}$ in the twoway relaying system under the scenario with integer fading

\footnotetext{
${ }^{1}$ For the general scenario with arbitrary Nakagami- $m$ fading parameters, to the best of the authors' knowledge, no exact outage probability expressions were ever reported. Furthermore, even for the particular scenario with integer fading parameters, due to the high complexity of the CDFs (such as (36) and (38) below) of the received SNRs, no exact ASEP or achievable data rate expressions were reported in the open literature.
}

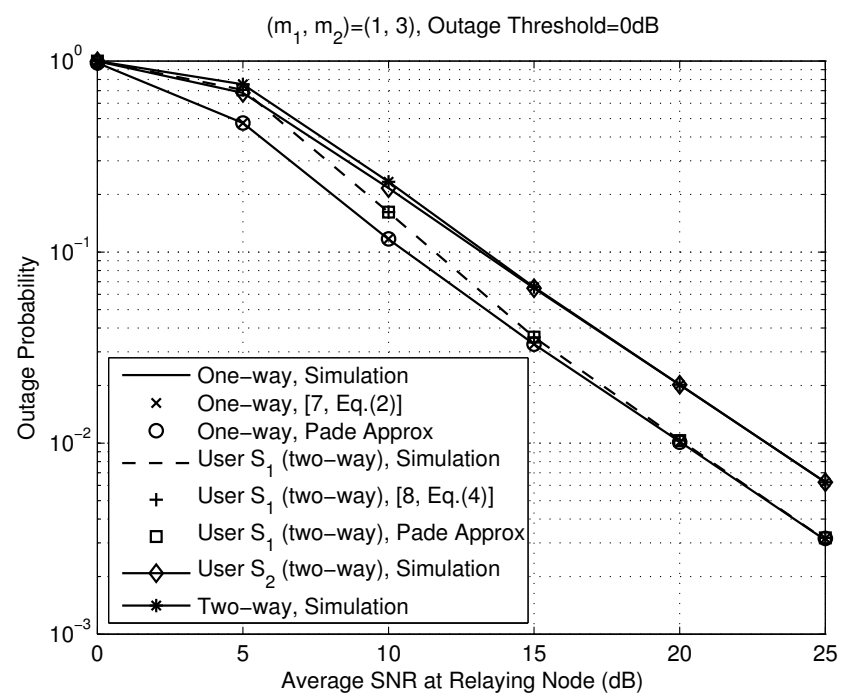

Fig. 3. Outage probability of one-way and two-way AF relaying systems over integer Nakagami- $m$ fading channels.

parameters $m_{1}$ and $m_{2}$, the outage probability is given by Eq. (38) at the top of the next page [10, Eq. (4)]. Using a similar approach, the outage probability of user $\mathrm{S}_{2}$ can be readily obtained.

Figure 3 depicts the outage probability of one-way and twoway relaying systems as well as that for users $S_{1}$ and $S_{2}$, as a function of the average SNR in $\mathrm{dB}$ at the relaying node. In the simulations, the fading parameters are non-symmetric with integer values $\left(m_{1}, m_{2}\right)=(1,3)$ where $m_{1}<m_{2}$, and the outage threshold is set to $0 \mathrm{~dB}$. For the one-way relaying case, three different results of outage probability, namely, the simulation results, the exact numerical results using (36), and the Padé approximant computed by (30) as well as (24), are compared. It is observed that these results are in perfect agreement with each other. Similarly, for the outage probability of user $\mathrm{S}_{1}$ in the two-way relaying system, the simulation results, the exact numerical results of (38), and the Padé approximant coincide perfectly with each other.

On the other hand, from Fig. 3, it is observed that user $\mathrm{S}_{1}$ in the two-way system suffers from higher outage probability than the one-way system in the low and medium SNR regions, due to the lower received SNR. However, in the high SNR region, their outage probabilities are almost the same. This is because, when the channel fading at two consecutive hops are non-symmetric with $m_{2} \gg m_{1}$ (implying $\gamma_{2} \gg \gamma_{1}$ ), the limits of the received SNRs of user D in the one-way system and user $\mathrm{S}_{1}$ in the two-way system are identical. That is, $\lim _{\gamma_{2} \rightarrow \infty} \gamma_{\mathrm{D}}=\lim _{\gamma_{2} \rightarrow \infty} \frac{\gamma_{1} \gamma_{2}}{\gamma_{1}+\gamma_{2}}=\gamma_{1}$ and $\lim _{\gamma_{2} \rightarrow \infty} \gamma_{\mathrm{S} 1}=$ $\lim _{\gamma_{2} \rightarrow \infty} \frac{\gamma_{1} \gamma_{2}}{2 \gamma_{1}+\gamma_{2}}=\gamma_{1}$. Then, we can see that the outage probability of user $\mathrm{S}_{2}$ is always higher than that of user $\mathrm{S}_{1}$, due to the lower received SNR (cf. (26) where $\gamma_{\mathrm{S} 2}<\gamma_{\mathrm{S} 1}$ if $\gamma_{1}<\gamma_{2}$ ). Finally, Fig. 3 shows that the outage probability of two-way relaying system is the highest and it is almost the same as that of user $\mathrm{S}_{2}$ in the medium and high SNR regions, as expected according to (35).

Figure 4 shows the outage probability under the scenario with non-integer Nakagami fading parameters where $m_{1}>$ $m_{2}$, considering $\left(m_{1}, m_{2}\right)=(2.5,1.1)$. It is seen that, 


$$
P_{1 \text { way }}^{\text {exact }}\left(\gamma_{\mathrm{th}}\right)=2 \exp \left[-\left(\frac{m_{1}}{\bar{\gamma}_{1}}+\frac{m_{2}}{\bar{\gamma}_{2}}\right) \gamma_{\mathrm{th}}\right] \sum_{n=0}^{m_{1}-1} \sum_{k=0}^{m_{2}-1} \sum_{l=0}^{k} C(n, k, l) \gamma_{\mathrm{th}}^{m_{1}+k} K_{n-l+1}\left(2 \sqrt{\frac{m_{1} m_{2}}{\bar{\gamma}_{1} \bar{\gamma}_{2}}} \gamma_{\mathrm{th}}\right)
$$

$$
C(n, k, l) \triangleq\left(l ! n !(k-l) !\left(m_{1}-n-1\right) !\right)^{-1}\left(\frac{\bar{\gamma}_{1}}{m_{1}}\right)^{\frac{1}{2}\left(n-l+1-2 m_{1}\right)}\left(\frac{\bar{\gamma}_{2}}{m_{2}}\right)^{\frac{1}{2}(l-n-1-2 k)}
$$

$$
\begin{aligned}
P_{\mathrm{S}_{1}}^{\text {exact }}\left(\gamma_{\mathrm{th}}\right)= & 1-2\left(\frac{m_{1}}{\bar{\gamma}_{1}}\right)^{m_{1}} \frac{\left(m_{2}-1\right) !}{\Gamma\left(m_{1}\right) \Gamma\left(m_{2}\right)} \exp \left[-\left(\frac{m_{1}}{\bar{\gamma}_{1}}+\frac{2 m_{2}}{\bar{\gamma}_{2}}\right) \gamma_{\mathrm{th}}\right] \\
& \times \sum_{p=0}^{m_{1}-1} \sum_{q=0}^{m_{2}-1} \sum_{k=0}^{q} \frac{1}{q !}\left(\begin{array}{c}
q \\
k
\end{array}\right)\left(\begin{array}{c}
m_{1}-1 \\
p
\end{array}\right)\left(\frac{m_{1}}{\bar{\gamma}_{1}}\right)^{-\frac{1}{2}(p-k+1)} \\
& \times \gamma_{\mathrm{th}}^{m_{1}+q}\left(\frac{2 m_{2}}{\bar{\gamma}_{2}}\right)^{q+\frac{1}{2}(p-k+1)} K_{p-k+1}\left(2 \sqrt{\frac{2 m_{1} m_{2}}{\bar{\gamma}_{1} \bar{\gamma}_{2}}} \gamma_{\mathrm{th}}\right) .
\end{aligned}
$$

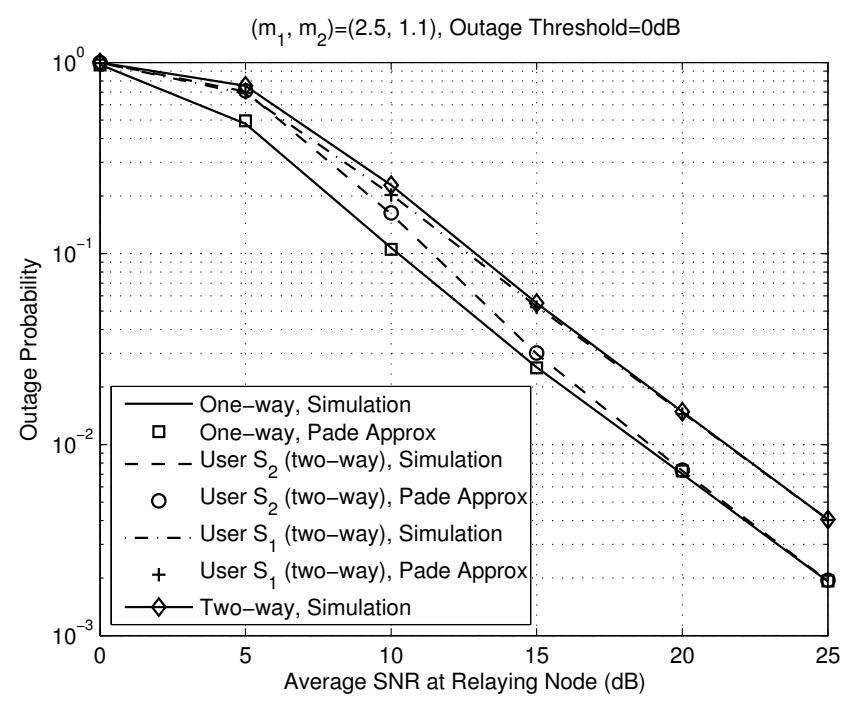

Fig. 4. Outage probability of one-way and two-way AF relaying systems over non-integer Nakagami- $m$ fading channels.

for either one-way relaying or users $S_{1}$ and $S_{2}$ in two-way relaying system, the numerical results of Padé approximant are perfectly consistent with the simulation results, which illustrates the effectiveness of the preceding analysis. On the other hand, it is observed that user $\mathrm{S}_{2}$ has lower outage probability than user $\mathrm{S}_{1}$, and the outage probability of the two-way system is dominated by that of user $\mathrm{S}_{1}$. Combining the observations in Figs. 3 and 4, we conclude that the outage probability of two-way relaying systems is dominated by the user whose channel fading is less severe (that is, the user associated with larger fading parameter). This conclusion is completely opposite to what one would expect. The reason behind lies in the fact that, if $m_{1} \gg m_{2}$ or equivalently $\gamma_{1} \gg \gamma_{2}$, we have $\lim _{\gamma_{1} \rightarrow \infty} \gamma_{\mathrm{S} 1}=\lim _{\gamma_{1} \rightarrow \infty} \frac{\gamma_{1} \gamma_{2}}{2 \gamma_{1}+\gamma_{2}}=\frac{1}{2} \gamma_{2}$ and $\lim _{\gamma_{1} \rightarrow \infty} \gamma_{\mathrm{S} 2}=\lim _{\gamma_{1} \rightarrow \infty} \frac{\gamma_{1} \gamma_{2}}{\gamma_{1}+2 \gamma_{2}}=\gamma_{2}$. Consequently, the outage probability of two-way relaying systems is dominated by user $\mathrm{S}_{1}$ who has lower received SNR.

\section{B. Average Symbol Error Probability}

Average symbol error probability (ASEP) is one of the most commonly used performance measures of wireless communication systems. By definition, ASEP is computed by integrating the conditional symbol error probability over the PDF of the received SNR. On the other hand, ASEP can be analyzed by using the MGF of the received SNR. Herein, we take the $M$-PSK constellation for instance since it is extensively adopted in real wireless systems, such as 3GPP long-term evolution (LTE) systems.

For the coherently detected $M$-PSK, with the MGF $\mathrm{M}_{\gamma_{j}}(s)$ of the received SNR, the ASEP of user $j, j \in\left\{\mathrm{D}, \mathrm{S}_{1}, \mathrm{~S}_{2}\right\}$ is given by [38, Eq. (8.23)]:

$$
\bar{P}_{j}=\frac{1}{\pi} \int_{0}^{\Theta} \mathrm{M}_{\gamma_{j}}\left(\frac{g_{\mathrm{PSK}}}{\sin ^{2} \theta}\right) \mathrm{d} \theta
$$

where $g_{\mathrm{PSK}}=\sin ^{2}(\pi / M)$ and $\Theta=(M-1) \pi / M$. Furthermore, an accurate approximation of the ASEP can be obtained by substituting the closed-form MGF given by (29) in conjunction with (11) into the following expression [39, Eq. (10)]:

$$
\begin{aligned}
\bar{P}_{j} \approx & \left(\frac{\Theta}{2 \pi}-\frac{1}{6}\right) \mathrm{M}_{\gamma_{j}}\left(g_{\mathrm{PSK}}\right)+\frac{1}{4} \mathrm{M}_{\gamma_{j}}\left(\frac{4}{3} g_{\mathrm{PSK}}\right) \\
& +\left(\frac{\Theta}{2 \pi}-\frac{1}{4}\right) \mathrm{M}_{\gamma_{j}}\left(\frac{g_{\mathrm{PSK}}}{\sin ^{2} \Theta}\right) .
\end{aligned}
$$

Therefore, applying (29) to (40), the ASEP of user $j, j \in$ $\left\{\mathrm{D}, \mathrm{S}_{1}, \mathrm{~S}_{2}\right\}$, can be given by

$$
\begin{aligned}
\bar{P}_{j} \approx & \left(\frac{\Theta}{2 \pi}-\frac{1}{6}\right) \mathrm{M}_{H_{w}}\left(\frac{g_{\mathrm{PSK}}}{a_{j}+b_{j}}\right) \\
& +\frac{1}{4} \mathrm{M}_{H_{w}}\left(\frac{4 g_{\mathrm{PSK}}}{3\left(a_{j}+b_{j}\right)}\right) \\
& +\left(\frac{\Theta}{2 \pi}-\frac{1}{4}\right) \mathrm{M}_{H_{w}}\left(\frac{g_{\mathrm{PSK}}}{\left(a_{j}+b_{j}\right) \sin ^{2} \Theta}\right) .
\end{aligned}
$$

Also, it is clear that the ASEP of one-way relaying system is equal to the ASEP of the destination $D$. On the other hand, the ASEP of two-way relaying system is defined as the mean 


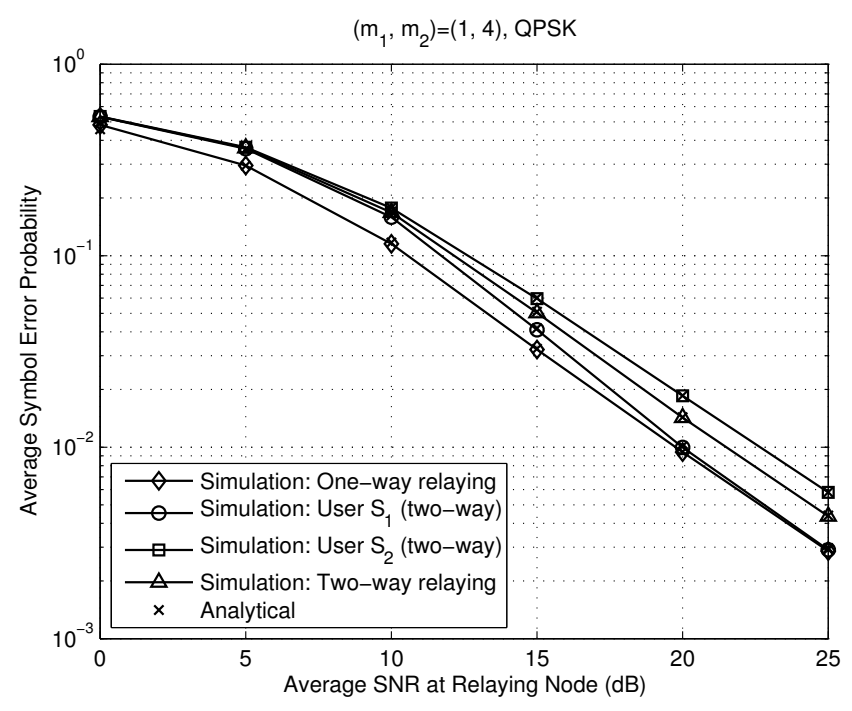

Fig. 5. Average symbol error probability of one-way and two-way AF relaying systems over non-symmetric fading channels.

of the ASEPs of users $\mathrm{S}_{1}$ and $\mathrm{S}_{2}$. In summary, the ASEPs of one-way and two-way relaying systems are given by

$$
\bar{P}_{1 \text { way }}=\bar{P}_{D}
$$

and

$$
\bar{P}_{2 \text { way }}=\frac{1}{2}\left(\bar{P}_{S 1}+\bar{P}_{S 2}\right),
$$

respectively.

Figure 5 compares the ASEPs of one-way and two-way relaying systems as a function of the average SNR in $\mathrm{dB}$ at the relaying node. Moreover, the ASEPs of users $\mathrm{S}_{1}$ and $\mathrm{S}_{2}$ in two-way relaying system are plotted in the same figure for comparison purposes. In the simulations, the channel fading parameters at two hops are non-symmetric with integer values $\left(m_{1}, m_{2}\right)=(1,4)$ and QPSK constellation is implemented. It is observed that the one-way relaying yields the lowest ASEP since it has the highest received SNR. For user $\mathrm{S}_{1}$ in twoway relaying system, higher ASEP is obtained, in comparison with one-way relaying in the low and medium SNR regions. However, in the high SNR region, they have almost the same ASEPs. On the other hand, the ASEP of user $\mathrm{S}_{2}$ in two-way system is always significantly higher than that of user $\mathrm{S}_{1}$. The reasons behind are similar to that for outage probability discussed in the preceding subsection. Furthermore, it is seen that the ASEP of two-way relaying is consistently higher than that of one-way relaying, and it lies between the ASEPs of users $S_{1}$ and $S_{2}$, as expected according to (43).

On the other hand, Fig. 6 compares the ASEPs of one-way and two-way relaying under symmetric fading channels (i.e. the Nakagami fading parameters at two consecutive hops are identical) with non-integer fading parameters $\left(m_{1}, m_{2}\right)=$ $(2.5,2.5)$. Clearly, when $m_{1}=m_{2}$, the received SNRs of users $\mathrm{S}_{1}$ and $\mathrm{S}_{2}$ are symmetric and, thus, their ASEPs are the same and identical to the ASEP of two-way relaying system. Therefore, unlike Fig. 5, Fig. 6 shows only the ASEPs of oneway and two-way systems while the ASEPs of users $S_{1}$ and $S_{2}$ are ignored. In this figure, it is seen that the ASEP of two-way relaying is always higher than that of one-way counterpart,

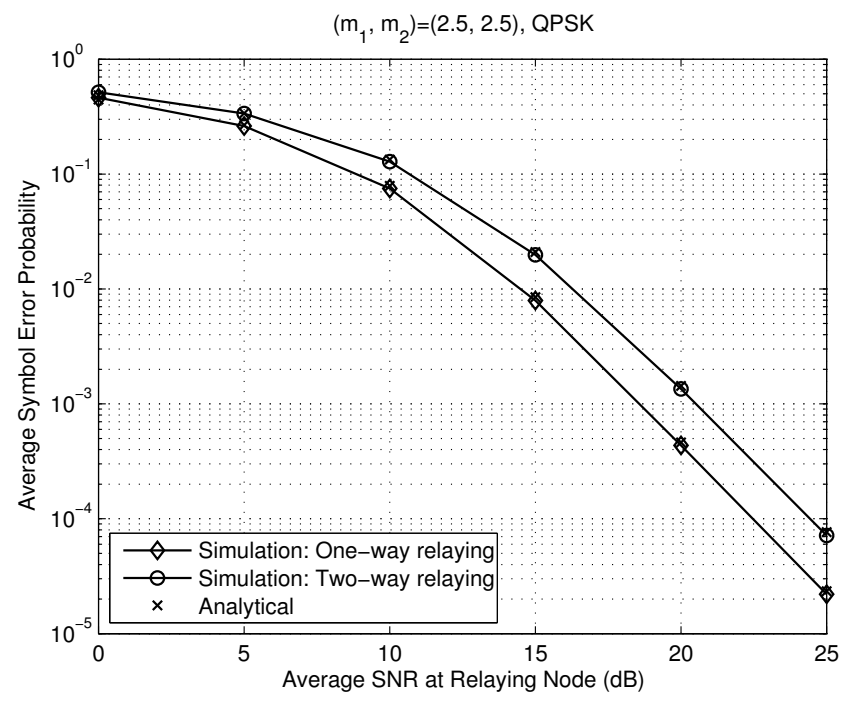

Fig. 6. Average symbol error probability of one-way and two-way AF relaying systems over symmetric fading channels.

due to its lower received SNR. As a final observation here, we note that from both Fig. 5 and Fig. 6, the analytical results coincide very well with the simulation results, demonstrating the effectiveness of our preceding analysis.

\section{Achievable Data Rate}

Based on the seminal Shannon theorem, the maximum achievable data rate of user $j, j \in\left\{\mathrm{D}, \mathrm{S}_{1}, \mathrm{~S}_{2}\right\}$, is given by

$$
\begin{aligned}
R_{j} & =\mathbb{E}\left\{\log _{2}\left(1+\gamma_{j}\right)\right\} \\
& \leq \log _{2}\left(1+\mathbb{E}\left\{\gamma_{j}\right\}\right),
\end{aligned}
$$

where we used the Jensen's inequality and the fact that the logarithm function is strictly concave to reach the inequality in (44), which implies that the first-order moment $\mathbb{E}\left\{\gamma_{j}\right\}$ given by (28) with $n=1$ can be exploited to obtain an upper bound on the achievable data rate. Furthermore, applying the Taylor series expansion to $\log _{2}\left(1+\gamma_{j}\right)$ with respect to $1+\mathbb{E}\left\{\gamma_{j}\right\}$, we get

$$
\begin{aligned}
R_{j}= & \frac{1}{\ln (2)} \mathbb{E}\left\{\sum_{n=0}^{\infty} \frac{\left(\gamma_{j}-\mathbb{E}\left\{\gamma_{j}\right\}\right)^{n}}{n !} \ln ^{(n)}\left(1+\mathbb{E}\left\{\gamma_{j}\right\}\right)\right\} \\
= & \frac{1}{\ln (2)} \ln \left(1+\mathbb{E}\left\{\gamma_{j}\right\}\right)-\frac{\mathbb{E}\left\{\gamma_{j}^{2}\right\}-\mathbb{E}^{2}\left\{\gamma_{j}\right\}}{2 \ln (2)\left(1+\mathbb{E}\left\{\gamma_{j}\right\}\right)^{2}} \\
& +O\left[\left(\gamma_{j}-\mathbb{E}\left\{\gamma_{j}\right\}\right)^{3}\right],
\end{aligned}
$$

where the moments $\mathbb{E}\left\{\gamma_{j}\right\}$ and $\mathbb{E}\left\{\gamma_{j}^{2}\right\}$ can be easily computed as per (28) as well as (7) and (8).

For the dual-hop AF relaying system under study, the achievable data rate is defined as the statistical mean of the instantaneous mutual information between the source and the destination, in the unit of bit/s/Hz. Specifically, for one-way relaying, since two transmission phases are involved for only one data transmission from the source to its destination, the achievable data rate is given by

$$
C_{1 \text { way }}=\frac{1}{2} R_{\mathrm{D}} \text {. }
$$


One the other hand, for two-way relaying, two users exchange data simultaneously during two transmission phases and, thus, the achievable data rate is given by [2, Eq. (20)]

$$
C_{2 \text { way }}=\frac{1}{2}\left(R_{\mathrm{S} 1}+R_{\mathrm{S} 2}\right) \text {. }
$$

Since computing $R_{j}, j \in\left\{\mathrm{D}, \mathrm{S}_{1}, \mathrm{~S}_{2}\right\}$, as per (45) involves only the first-order and second-order moments of the received SNR, the expressions given in (46) and (47) are referred to as the second-order moment approximation to the achievable data rate. Also, comparing (26) with (25) shows that $\gamma_{\mathrm{S}_{1}}, \gamma_{\mathrm{S}_{2}}<$ $\gamma_{\mathrm{D}}$. As such, the achievable data rate ratio of two-way relaying over one-way counterpart is upper bounded by

$$
\eta=\frac{C_{2 \text { way }}}{C_{1 \text { way }}}<2 .
$$

Notice that in appearance, (46) and (47) seem similar to each other. In essence, however, (47) reflects the inherent superiority of two-way relaying over its one-way counterpart in terms of achievable data rate. That is, the technique of two-way relaying is able to double the achievable data rate compared to one-way relaying while using the same amount of system resources. This is indeed the starting point of the idea of two-way relaying originally proposed in [2]. Nevertheless, there is no definite answer in the open literature to the following important question: what conditions must be met in order to double the achievable data rate? The proposed framework enables to answer this question in a simple and unified way. Specifically, the following results show that only when the SNR is very high can two-way relaying double the achievable data rate compared to one-way relaying for the same channel conditions, i.e. $\eta$ in (48) approaches its upper bound 2 .

The accuracy of (46)-(47) is examined in Fig. 7, in comparison with simulation results. This figure shows the achievable data rate of one-way/two-way relaying versus the average SNR in $\mathrm{dB}$ at the relaying node, considering integer or noninteger Nakagami- $m$ fading parameters. It is observed that the numerical results of achievable data rate given by (45) coincide very well with the simulation results. Furthermore, two-way relaying always achieves higher achievable data rate than the one-way relaying, since the simultaneous transmissions by the two users in the former benefits improving spectral efficiency significantly.

In order to gain more insights into the relationship of achievable data rate between one-way and two-way relaying systems, Fig. 8 depicts the ratio of the achievable data rate of two-way relaying to that of its one-way counterpart, based on the same simulation settings as Fig. 7. It is observed that the data-rate ratio improves with increasing average SNR and it approaches the upper bound (48) at high SNR. Furthermore, compared to the case with $\left(m_{1}, m_{2}\right)=(1,2)$, the slightly steeper slope of the curve pertaining to the case where $\left(m_{1}, m_{2}\right)=(4.2,3.5)$ implies that the gain of data-rate ratio improves when the values of channel fading parameters become larger, thus, the intersection of both curves in Fig. 8 . However, it is observed that the improvement is not significant even if the values of the fading parameters vary a lot. In conclusion, the superiority of two-way relaying over one-way

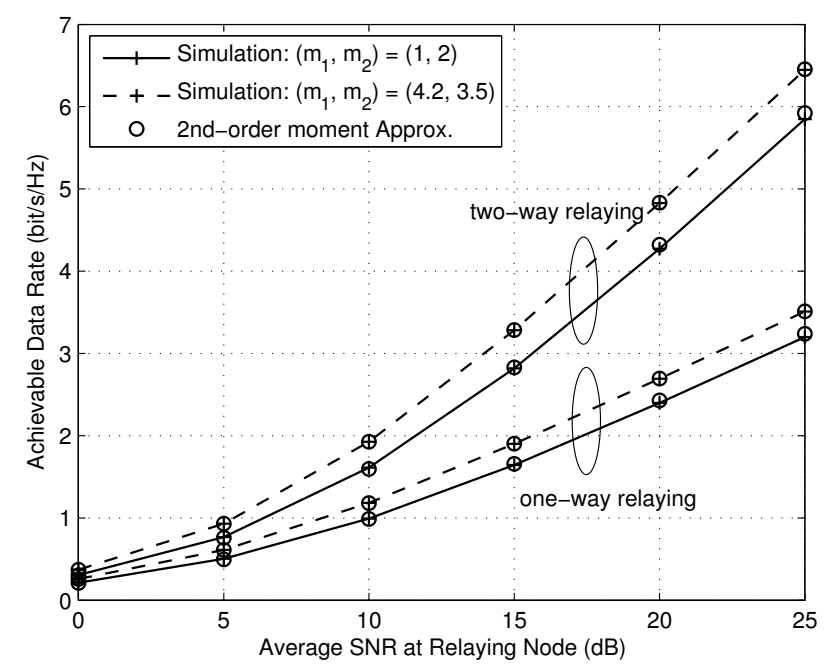

Fig. 7. Achievable data rate of one-way and two-way AF relaying systems.

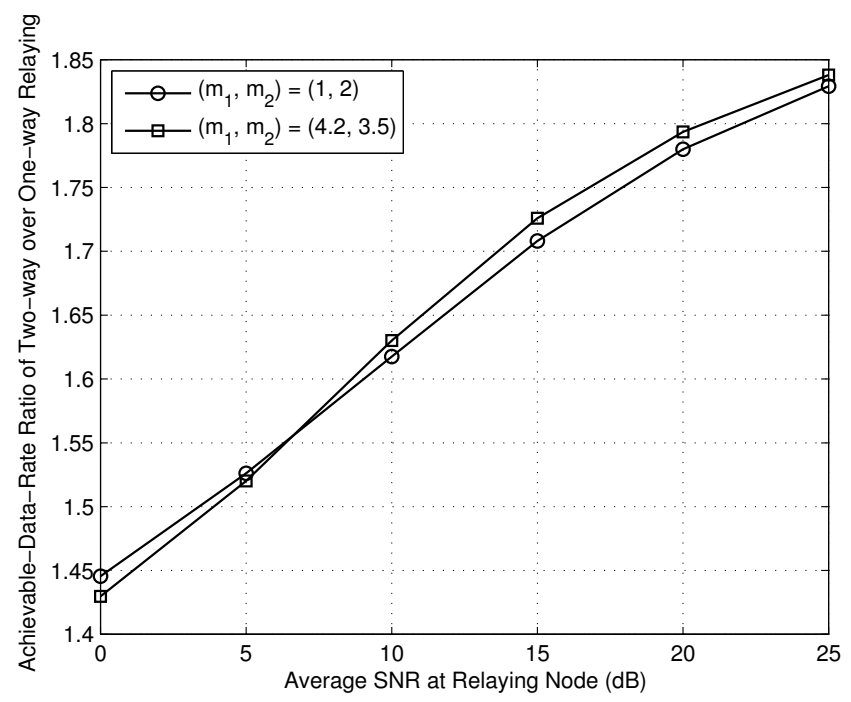

Fig. 8. The ratio of the achievable data rate of two-way relaying to that of its one-way counterpart.

relaying in terms of achievable data rate becomes significant in the high SNR region, and it is slightly influenced by the values of channel fading parameters.

Remark 5: The technique of the second-order moment approximation to achievable data rate was also demonstrated to be highly effective in one-way semi-blind AF relaying systems [14], [23]. On the other hand, a unified MGF-based approach for computing the achievable data rate over general fading channels was recently developed in [40, Eq. (7)]. Unfortunately, even with the simple Padé approximant (23) to the MGF, the unified MGF-based approach cannot be applied here and it is expected that no closed-form expression pertaining to achievable data rate can be derived in terms of common special functions.

\section{CONCLUSIONS}

In this paper, a unified framework was developed to analyze and compare system performance of one-way and two-way 
CSI-assisted AF relaying over arbitrary Nakagami- $m$ fading channels. The framework is rooted in the moments of the weighted harmonic mean of two Gamma variables and the theory of Padé approximants. Our results reveal that the superiority of two-way relaying over its one-way counterpart in terms of achievable data rate becomes overwhelming in the high SNR region, and that it is slightly influenced by the values of channel fading parameters. However, two-way relaying suffers from higher outage probability and ASEP than its one-way counterpart in the whole SNR range under consideration. In addition, the outage probability and ASEP of two-way relaying are dominated by the user whose channel fading is less severe. The proposed framework and resultant performance analysis benefit the community understanding and comparing one-way with two-way relaying over general fading channels in a simple and unified way. Furthermore, the framework can be extended to other related areas, such as the dual-hop relaying scenario with optimal power allocation and the scenario of multi-hop relaying.

\section{APPENDIX A}

\section{PROOF OF THEOREM 1}

Let $X_{1}$ and $X_{2}$ be two i.n.i.d random variables distributed as $X_{i} \sim G\left(\alpha_{i}, \beta_{i}\right), i=1,2$. Then, by the definition (1), the $n^{\text {th }}$-order moment of the weighted harmonic mean of $X_{1}$ and $X_{2}$ can be computed as Eqs. (49)-(51) at the top of the next page, where we used [27, vol. 1, Eq. (2.3.6.9)] to derive (51), with $\Psi(a, b ; x)$ being the Tricomi confluent hypergeometric function [22, Eq. (9.210.2)].

Then, substituting the PDF (3) into (51), we obtain Eqs. (52)-(53) at the top of the next page. Subsequently, applying [22, Eq. (7.621.6)] to (53) yields the following expressions:

1) Case I $\left(b \beta_{1} \leq a \beta_{2}\right)$ : The $n^{\text {th }}$-order moment of $H_{w}$ is given by Eq. (54) in the middle of the next page.

2) Case II $\left(b \beta_{1}>a \beta_{2}\right)$ : The $n^{\text {th }}$-order moment of $H_{w}$ is given by Eq. (55) in the middle of the next page.

Finally, combining (54) with (55) and performing some algebraic manipulations lead to the intended (4).

\section{APPENDIX B \\ PROOF OF THEOREM 2}

By virtue of the definition (1), in order to obtain the MGF of the weighted harmonic mean of $X_{1}$ and $X_{2}$, we first derive the MGFs of its components $\frac{a}{(a+b) X_{1}}$ and $\frac{b}{(a+b) X_{2}}$. Based on the PDF of $X_{1}$ shown in (3), the MGF of $\frac{a}{(a+b) X_{1}}$ is obtained as Eq. (56) at the bottom of the next page, where we used [22, Eq. (3.471.9)] to derive (56).

Similarly, the MGF of $\frac{b}{(a+b) X_{2}}$ can be derived and it is expressed as

$$
\begin{aligned}
\mathrm{M}_{2}(s)= & \frac{2}{\Gamma\left(\alpha_{2}\right)}\left(\frac{b s}{(a+b) \beta_{2}}\right)^{\frac{1}{2} \alpha_{2}} \\
& \times K_{\alpha_{2}}\left(2 \sqrt{\frac{b s}{(a+b) \beta_{2}}}\right) .
\end{aligned}
$$

Then based on (56)-(57) and using [29, Eq. (7)], after some algebraic manipulations, the MGF of the weighted harmonic mean of $X_{1}$ and $X_{2}$ can be given by Eq. (58) at the bottom of the next page, where the constants $k_{1}$ and $k_{2}$ were previously shown in (12), $J_{1}(x)$ denotes the first-order Bessel function of the first kind [22, Eq. (8.402)] and $K_{v}(x)$ is the $v^{\text {th }}$ order modified Bessel function of the second kind [22, Eq. (8.432.6)]. Subsequently, applying [27, vol. 2, Eq. (2.16.43.1)] to (58) and performing some algebraic manipulations yield the desired (11).

\section{REFERENCES}

[1] J. N. Laneman, D. N. C. Tse, and G. W. Wornell, "Cooperative diversity in wireless networks: efficient protocols and outage behaviour," IEEE Trans. Inf. Theory, vol. 50, no. 12, pp. 3062-3080, Dec. 2004.

[2] B. Rankov and A. Wittneben, "Spectral efficient protocols for halfduplex fading relaying channels," IEEE J. Sel. Areas Commun., vol. 25, no. 2, pp. 373-389, Feb. 2007.

[3] M. O. Hasna and M.-S. Alouini, "End-to-end performance of transmission systems with relays over Rayleigh-fading channels," IEEE Trans. Wireless Commun., vol. 2, no. 6, pp. 1126-1131, Feb. 2003.

[4] D. B. da Costa and S. Aïssa, "Performance analysis of relay selection techniques with clustered fixed-gain relays," IEEE Signal Process. Lett., vol. 17, no. 2, pp. 201-204, Feb. 2010.

[5] R. H. Y. Louie, Y. Li, and B. Vucetic, "Practical physical layer network coding for two-way relay channels: performance analysis and comparison," IEEE Trans. Wireless Commun., vol. 9, no. 2, pp. 764-777, Feb. 2010.

[6] S. Ikki and S. Aïssa, "Performance analysis of two-way amplify-andforward relaying in the presence of co-channel interferences," IEEE Trans. Commun., vol. 60, no. 4, pp. 933-939, Apr. 2012.

[7] M. Xia and S. Aïssa, "Cooperative AF relaying in spectrum-sharing systems: performance analysis under average interference power constraints and Nakagami- $m$ fading," IEEE Trans. Commun., to appear.

[8] N. Yang, M. Elkashlan, and J. Yuan, "Outage probability of multiuser relay networks in Nakagami- $m$ fading channels," IEEE Trans. Veh. Technol., vol. 59, no. 5, pp. 2120-2132, June 2010.

[9] D. Senaratne and C. Tellambura, "Unified exact performance analysis of two-hop amplify-and-forward relaying in Nakagami- $m$ fading," IEEE Trans. Veh. Technol., vol. 59, no. 3, pp. 1529-1533, Mar. 2010.

[10] J. Yang, P. Fan, T. Q. Duong, and X. Lei, "Exact performance of twoway AF relaying in Nakagami- $m$ fading channels," IEEE Trans. Wireless Commun., vol. 10, no. 3, pp. 980-987, Mar. 2011.

[11] P. K. Upadhyay and S. Prakriya, "Performance of two-way opportunistic relaying with analog network coding over Nakagami- $m$ fading," IEEE Trans. Veh. Technol., vol. 60, no. 4, pp. 1965-1971, Apr. 2011.

[12] L. Rubio, J. Reig, and N. Cardona, "Evaluation of Nakagami fading behaviour based on measurements in urban scenarios," ELSEVIER Int. J. Electron. Commun., vol. 61, no. 2, pp. 135-138, Feb. 2007.

[13] M. D. Yacoub, "The $\kappa-\mu$ distribution and the $\eta-\mu$ distribution," IEEE Antennas Propag. Mag., vol. 49, no. 1, pp. 68-81, Feb. 2007.

[14] M. Xia, C. Xing, Y.-C. Wu, and S. Aïssa, "Exact performance analysis of dual-hop semi-blind AF relaying over arbitrary Nakagami- $m$ fading channels," IEEE Trans. Wireless. Commun., vol. 10, no. 10, pp. 34493459, Oct. 2011.

[15] M. O. Hasna and M.-S. Alouini, "Harmonic mean and end-to-end performance of transmission systems with relays," IEEE Trans. Commun., vol. 52, no. 1, pp. 130-135, Jan. 2004.

[16] L. L. Yang and H.-H. Chen, "Error probability of digital communications using relay diversity over Nakagami- $m$ fading channels," IEEE Trans. Wireless Commun., vol. 7, no. 5, pp. 1806-1811, May 2008.

[17] N. C. Beaulieu and Y. Chen, "An accurate approximation to the average error probability of cooperative diversity in Nakagami- $m$ fading," IEEE Trans. Wireless Commun., vol. 9, no. 9, pp. 2707-2711, Sep. 2010.

[18] Y. Han, S. H. Ting, C. K. Ho, and W. H. Chin, "Performance bounds for two-way amplify-and-forward relaying," IEEE Trans. Wireless Commun., vol. 8, no. 1, pp. 432-439, Jan. 2009.

[19] P. S. Bullen, A Dictionary of Inequalities, Chapman and Hall/CRC Press, 1998.

[20] F. W. J. Olver, NIST Handbook of Mathematical Functions, Cambridge University Press, 2010.

[21] N. Balakrishnan and V. B. Nevzorov, A Primer on Statistical Distributions, John Wiley \& Sons Inc., 2003.

[22] I. S. Gradshteyn and I. M. Ryzhik, Table of Integrals, Series and Products, 7th Ed., Academic Press, 2007.

[23] D. B. da Costa and S. Aïssa, "Capacity analysis of cooperative systems with relay selection in Nakagami- $m$ fading", IEEE Commun. Lett., vol. 13, no. 9, pp. 637-639, Sep. 2009. 


$$
\begin{aligned}
\mathbb{E}\left\{H_{w}^{n}\right\} & =(a+b)^{n} \int_{0}^{\infty} \int_{0}^{\infty}\left(\frac{x_{1} x_{2}}{a x_{2}+b x_{1}}\right)^{n} f_{X_{1}}\left(x_{1}\right) f_{X_{2}}\left(x_{2}\right) \mathrm{d} x_{1} \mathrm{~d} x_{2} \\
& =\frac{(a+b)^{n}}{b^{n}} \int_{0}^{\infty}\left[\int_{0}^{\infty} \frac{x_{1}^{n}}{\left(x_{1}+\frac{a}{b} x_{2}\right)^{n}} f_{X_{1}}\left(x_{1}\right) \mathrm{d} x_{1}\right] x_{2}^{n} f_{X_{2}}\left(x_{2}\right) \mathrm{d} x_{2} \\
& =\frac{(a+b)^{n}}{b^{n} \beta_{1}^{\alpha_{1}} \Gamma\left(\alpha_{1}\right)} \int_{0}^{\infty}\left[\int_{0}^{\infty} \frac{x_{1}^{n+\alpha_{1}-1}}{\left(x_{1}+\frac{a}{b} x_{2}\right)^{n}} e^{-\frac{x_{1}}{\beta_{1}}} \mathrm{~d} x_{1}\right] x_{2}^{n} f_{X_{2}}\left(x_{2}\right) \mathrm{d} x_{2} \\
& =\left(\frac{a}{b \beta_{1}}\right)^{\alpha_{1}} \frac{(a+b)^{n} \Gamma\left(n+\alpha_{1}\right)}{b^{n} \Gamma\left(\alpha_{1}\right)} \int_{0}^{\infty} x_{2}^{n+\alpha_{1}} \Psi\left(n+\alpha_{1}, \alpha_{1}+1, \frac{a}{b \beta_{1}} x_{2}\right) f_{X_{2}}\left(x_{2}\right) \mathrm{d} x_{2} .
\end{aligned}
$$

$$
\begin{aligned}
\mathbb{E}\left\{H_{w}^{n}\right\} & =\left(\frac{a}{b \beta_{1}}\right)^{\alpha_{1}} \frac{(a+b)^{n} \Gamma\left(n+\alpha_{1}\right)}{b^{n} \beta_{2}^{\alpha_{2}} \Gamma\left(\alpha_{1}\right) \Gamma\left(\alpha_{2}\right)} \int_{0}^{\infty} x_{2}^{n+\alpha_{1}+\alpha_{2}-1} \Psi\left(n+\alpha_{1}, \alpha_{1}+1, \frac{a}{b \beta_{1}} x_{2}\right) e^{-\frac{x_{2}}{\beta_{2}}} \mathrm{~d} x_{2} \\
& =\frac{(a+b)^{n} \Gamma\left(n+\alpha_{1}\right)}{\Gamma\left(\alpha_{1}\right) \Gamma\left(\alpha_{2}\right)}\left(\frac{\beta_{1}}{a}\right)^{n}\left(\frac{b \beta_{1}}{a \beta_{2}}\right)^{\alpha_{2}} \int_{0}^{\infty} x^{n+\alpha_{1}+\alpha_{2}-1} \Psi\left(n+\alpha_{1}, \alpha_{1}+1, x\right) e^{-\frac{b \beta_{1}}{a \beta_{2}} x} \mathrm{~d} x
\end{aligned}
$$

$$
\begin{aligned}
\mathbb{E}\left\{H_{w}^{n}\right\}= & B\left(n+\alpha_{1}, n+\alpha_{2}\right) \frac{(a+b)^{n} \Gamma\left(n+\alpha_{1}+\alpha_{2}\right)}{\Gamma\left(\alpha_{1}\right) \Gamma\left(\alpha_{2}\right)}\left(\frac{\beta_{1}}{a}\right)^{n}\left(\frac{b \beta_{1}}{a \beta_{2}}\right)^{\alpha_{2}} \\
& \times_{2} F_{1}\left(n+\alpha_{2}, n+\alpha_{1}+\alpha_{2} ; 2 n+\alpha_{1}+\alpha_{2} ; 1-\frac{b \beta_{1}}{a \beta_{2}}\right) .
\end{aligned}
$$

$$
\begin{aligned}
\mathbb{E}\left\{H_{w}^{n}\right\}= & B\left(n+\alpha_{1}, n+\alpha_{2}\right) \frac{(a+b)^{n} \Gamma\left(n+\alpha_{1}+\alpha_{2}\right)}{\Gamma\left(\alpha_{1}\right) \Gamma\left(\alpha_{2}\right)}\left(\frac{\beta_{2}}{b}\right)^{n}\left(\frac{a \beta_{2}}{b \beta_{1}}\right)^{\alpha_{1}} \\
& \times{ }_{2} F_{1}\left(n+\alpha_{1}, n+\alpha_{1}+\alpha_{2} ; 2 n+\alpha_{1}+\alpha_{2} ; 1-\frac{a \beta_{2}}{b \beta_{1}}\right) .
\end{aligned}
$$

$$
\begin{aligned}
\mathrm{M}_{1}(s) & =\frac{1}{\beta_{1}^{\alpha_{1}} \Gamma\left(\alpha_{1}\right)} \int_{0}^{\infty} \exp \left(\frac{-a s}{(a+b) x}\right) x^{\alpha_{1}-1} \exp \left(-\frac{x}{\beta_{1}}\right) \mathrm{d} x \\
& =\frac{1}{\beta_{1}^{\alpha_{1}} \Gamma\left(\alpha_{1}\right)} \int_{0}^{\infty} x^{\alpha_{1}-1} \exp \left[\frac{-a s}{(a+b) x}-\frac{x}{\beta_{1}}\right] \mathrm{d} x \\
& =\frac{2}{\Gamma\left(\alpha_{1}\right)}\left(\frac{a s}{(a+b) \beta_{1}}\right)^{\frac{1}{2} \alpha_{1}} K_{\alpha_{1}}\left(2 \sqrt{\frac{a s}{(a+b) \beta_{1}}}\right)
\end{aligned}
$$

$$
\mathrm{M}_{H_{w}}(s)=1-\frac{8 k_{1}^{\alpha_{1}} k_{2}^{\alpha_{2}}}{\Gamma\left(\alpha_{1}\right) \Gamma\left(\alpha_{2}\right)} \sqrt{s} \int_{0}^{\infty} x^{\alpha_{1}+\alpha_{2}} J_{1}(2 \sqrt{s} x) K_{\alpha_{1}}\left(2 k_{1} x\right) K_{\alpha_{2}}\left(2 k_{2} x\right) \mathrm{d} x
$$

[24] A. Stuart and J. K. Ord, Kendall's Advanced Theory of Statistics, Vol. 1: Distribution Theory, 6th Ed., Hodder Arnold Pub., 1994.

[25] R. Kwan and C. Leung, "On the applicability of the Pearson method for approximating distributions in wireless communications," IEEE Trans. Commun., vol. 55, no. 11, pp. 2065-2069, Nov. 2007.

[26] M. D. Springer, The Algebra of Random Variables, John Wiley \& Sons Inc., 1979.

[27] A. P. Prudnikov, Y. A. Brychkov, and O. I. Marichev, Integrals and Series, Gordon and Breach Science Publishers, 1986.

[28] G. A. Baker, Jr. and P. Graves-Morris, Padé Approximants, 2nd Ed., Cambridge University Press, 1996.

[29] V. Asghari, A. Maaref, and S. Aïssa, "Symbol error probability analysis for multihop relaying over Nakagami fading channels," in Proc. IEEE WCNC'10, Sydney, Australia, pp. 1-5, Apr. 2010.
[30] F. Johansson et al, "MPMATH: a Python library for arbitraryprecision floating-point arithmetic (version 0.14)," Feb. 2010, Available: http://code.google.com/p/mpmath/.

[31] Y. L. Luke, The Special Functions and Their Approximations, vol. 2, Academic Press, 1969.

[32] H. Amindavar and J. A. Ritcey, "Padé approximations of probability density functions," IEEE Trans. Aerosp. Elect. Syst., vol. 30, no. 2, pp. 416-424, Apr. 1994.

[33] G. K. Karagiannidis, "Moments-based approach to the performance analysis of equal gain diversity in Nakagami- $m$ fading," IEEE Trans. Commun., vol. 52, no. 5, pp. 685-690, May 2004.

[34] G. K. Karagiannidis, "Performance bounds of multi-hop wireless communications with blind relays over generalized fading channels," IEEE Trans. Wireless Commun., vol. 5, no. 3, pp. 498-503, Mar. 2006. 
[35] M. H. Ismail and M. M. Matalgah, "Performance of dual maximal ratio combining diversity in nonidentical correlated Weibull fading channels using Padé approximation," IEEE Trans. Commun., vol. 54, no. 3, pp. 397-402, Mar. 2006.

[36] M. Di Renzo, F. Graziosi, and F. Santucci, "A framework for the analysis of UWB receivers in sparse multipath channels with intra-pulse interference via Padé expansion," IEEE Trans. Commun., vol. 56, no. 4 , pp. 535-541, Apr. 2008.

[37] G. L. Stüber, Principles of Mobile Communication, 2nd Ed., Kluwer Academic Publishers, 2002.

[38] M. K. Simon and M.-S. Alouini, Digital Communication over Fading Channels, 2nd Ed., John Wiley \& Sons Inc., 2005.

[39] M. R. McKay, A. Zanella, I. B. Collings, and M. Chiani, "Error probability and SINR analysis of optimum combining in Rician fading," IEEE Trans. Commun., vol. 57, no. 3, pp. 676-687, Mar. 2009.

[40] M. Di Renzo, F. Graziosi, and F. Santucci, "Channel capacity over generalized fading channels: a novel MGF-based approach for performance analysis and design of wireless communication systems," IEEE Trans. Veh. Technol., vol. 59, no. 1, pp. 127-149, Jan. 2010.

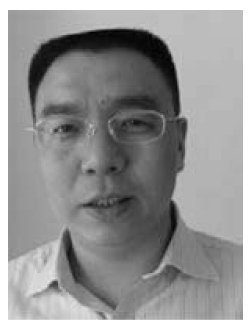

Minghua Xia (M'12) obtained his Ph.D. degree in Telecommunications and Information Systems from Sun Yat-sen University, Guangzhou, China, in 2007. From Mar. 2007 to July 2009, he was with the Electronics and Telecommunications Research Institute (ETRI) of South Korea, Beijing R\&D Center, Beijing, China, where he worked as a member of engineering staff and participated in the projects on the physical layer design of 3GPP LTE mobile communications. From Aug. 2009 to Feb. 2011, he was with The University of Hong Kong (HKU), Hong Kong, as a Postdoctoral Fellow. Currently, he is with King Abdullah University of Science and Technology (KAUST), Saudi Arabia. His research interests are in the area of network information theory and space-time signal processing, and in particular the design and performance analysis of multiuser multi-antenna systems, cooperative relaying systems, and cognitive radio networks.

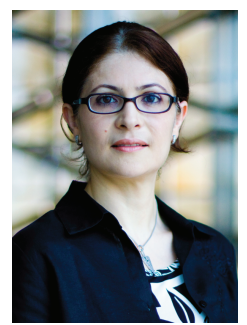

Sonia Aïssa (S'93-M'00-SM'03) received her Ph.D. degree in Electrical and Computer Engineering from McGill University, Montreal, QC, Canada, in 1998. Since then, she has been with the National Institute of Scientific Research-Energy, Materials, and Telecommunications (INRS-EMT), University of Quebec, Montreal, QC, Canada, where she is a Full Professor.

From 1996 to 1997, she was a Researcher with the Department of Electronics and Communications of Kyoto University, Kyoto, Japan, and with the Wireless Systems Laboratories of NTT, Kanagawa, Japan. From 1998 to 2000, she was a Research Associate at INRS-EMT, Montreal. From 2000 to 2002, while she was an Assistant Professor, she was a Principal Investigator in the major program of personal and mobile communications of the Canadian Institute for Telecommunications Research (CITR), leading research in radio resource management for code division multiple access systems. From 2004 to 2007, she was an Adjunct Professor with Concordia University, Montreal. In 2006, she was Visiting Invited Professor with the Graduate School of Informatics, Kyoto University, Kyoto, Japan. Her research interests lie in the area of wireless and mobile communications, and include radio resource management, cross-layer design and optimization, design and analysis of multiple antenna (MIMO) systems, cognitive and cooperative transmission techniques, and performance evaluation, with a focus on Cellular, Ad Hoc, and Cognitive Radio networks.

Dr. Aïssa was the Founding Chair of the Montreal Chapter IEEE Women in Engineering Society in 2004-2007, acted or is currently acting as Technical Program Leading Chair or Cochair for the Wireless Communications Symposium of the IEEE International Conference on Communications (ICC) in 2006, 2009, 2011 and 2012, as PHY/MAC Program Chair for the 2007 IEEE Wireless Communications and Networking Conference (WCNC), and as Technical Program Committee Cochair of the 2013 IEEE Vehicular Technology Conference - spring (VTC). She has served as a Guest Editor of the EURASIP Journal on Wireless Communications and Networking in 2006, and as Associate Editor of the IEEE WIRELESS COMMUNICATIONS MAGAZINE in 20062010. She is currently an Editor of the IEEE TRANSACTIONS ON WIRELESS COMMUNICATIONS, the IEEE TRANSACTIONS ON COMMUNICATIONS and the IEEE COMmunications Magazine, and Associate Editor of the Wiley Security and Communication Networks Journal. Awards and distinctions to her credit include the Quebec Government FQRNT Strategic Fellowship for Professors-Researchers in 2001-2006; the INRS-EMT Performance Award in 2004 and 2011 for outstanding achievements in research, teaching and service; the IEEE Communications Society Certificate of Appreciation in 2006-2012; and the Technical Community Service Award from the FQRNT Center for Advanced Systems and Technologies in Communications (SYTACom) in 2007. She is also co-recipient of Best Paper Awards from IEEE ISCC 2009, WPMC 2010, IEEE WCNC 2010, IEEE ICCIT 2011 and IEEE VTC 2011 (Kansai Section); and recipient of NSERC (Natural Sciences and Engineering Research Council of Canada) Discovery Accelerator Supplement Award. 